\title{
Utilizing an Open-Source Workflow for the Analysis of Atom Dynamics in Two- Dimensional Materials
}

EN O’Connell ${ }^{1 *}$, M Hennessy $^{1}$, E Courtney ${ }^{1}$, QM Ramasse ${ }^{2,3}$, B Kardynal ${ }^{4}$, H Hofsaess ${ }^{5}$, M Ghorbani$\mathrm{Asl}^{6}$, A Krasheninnikov ${ }^{6,7}$ and U Bangert ${ }^{1}$

1. Dept. of Physics, School of Natural Sciences \& Bernal Institute, University of Limerick, Limerick, Ireland.

2. SuperSTEM Laboratory, SciTech Daresbury Campus, Keckwick Lane, Daresbury, UK.

3. School of Physics and School of Chemical and Process Engineering, University of Leeds, Leeds, UK.

4. Peter Grünberg Institute, Forschungszentrum Jülich, Germany.

5. Physikalisches Institut, Georg-August-Universität Göttingen, Göttingen, Germany.

6. Helmholtz-Zentrum Dresden-Rossendorf, Institute of Ion Beam Physics and Materials Research, Dresden, Germany.

7. Department of Applied Physics, Aalto University School of Science, Aalto, Finland.

*Corresponding author: eoghan.oconnell@ul.ie

In the previous decade, open-course python packages, such as Hyperspy [1], have gifted researchers in the field of electron microscopy (EM) with easy to use, well-documented, and most-importantly flexible analysis tools. Although there is a small initial barrier for those not versed in programming and scripting, the simplicity and depth of documentation of these python packages allow many to contribute to their development. The power of these programs is the ability to adapt and create code for many areas of data and image analysis. Combining python-based packages developed for EM and material science such as Hyperspy [2], RigidRegistration [3], Atomap [4], PyPrismatic [5,6] and Atomic Simulation Environment (ASE) [7] allows for automated and reproducible image analysis.

Accurately controlling the structure and properties of two-dimensional (2D) materials via doping is a vital step in the development of 2D-based devices [8,9]. Ion-implantation is one such doping method, successfully implemented with graphene [10,11] and $\mathrm{MoS}_{2}$ [12]. This top-down approach is clean and controlled, with high source purity and has been used widely in industry in the past fifty years [13]. Using the above open-source tools, we can understand the physical and opto-electronic properties of such doped materials (e.g., $\mathrm{MoS}_{2}+\mathrm{Se} / \mathrm{Au}$ ) directly with high angle annular dark field scanning transmission EM (HAADF STEM) and electron energy loss spectroscopy (EELS). The position and configuration of lattice and dopant atoms and adatoms can be verified, as well as electron beam and doping induced effects, such as hole formation, defects, dislocations and contamination.

A serious limitation of imaging monolayer materials is electron beam induced damage. We attempt, with a low voltage $60 \mathrm{keV}$ electron beam, to understand the beam-sample interaction at atomic resolution by varying the electron dose rate. At low dose rates, single frame image contrast becomes a problem. Opensource image registration programs, such as RigidRegistration, allow for accurate alignment of subsequent images in the series, while atom fitting programs such as Atomap allow for the creation of atomic models via different HAADF intensities [14]. These models can quickly be simulated in PyPrismatic, a fast image simulation program, and compared iteratively to the raw or filtered experimental data, similar to [15]. This process is summarised in Figure 1. Electronic, energetic and structural properties of these models can then be analysed using ASE or an external tool, such as a density functional theory (DFT) software. An additional challenge when imaging such materials is 
contamination, which can have a large effect on the resulting image analysis workflow summarised in Figure 1 (a). Unlike the high $\mathrm{Z}$ nanoparticles described and analysed by Jones et al [15], carbon-based contamination has a large effect on the visibility and quantifiability of chalcogens, especially sulphur, in monolayers. Figure 1(b) shows an example experimental image of Se implanted $\mathrm{MoS}_{2}$ being fed into the workflow from (a), creating a model and image simulation for further analysis. With this method, we investigate monolayer samples with image series of up to 350 frames.

Using python packages to analyse experimentally imaged atomic structures is not only possible, but quick, flexible and reproducible. We limit the electron-beam effects on monolayers by varying the dose rate in a $60 \mathrm{keV}$ STEM, and by combining several open-source python packages, we analyse the properties of ion-implanted 2D materials at atomic resolution [16].

\section{References:}

[1] F de la Pena et al., Microsc. Microanal. 23 (2017), p. 214.

[2] F de la Peña et al., doi:10.5281/zenodo.592838 (2018).

[3] BH Savitzky et al., Ultramicroscopy 191 (2018), p. 56.

[4] M Nord et al., Adv. Struct. Chem. Imaging 3 (2017), p. 9.

[5] A Pryor, C Ophus and J Miao, Adv. Struct. Chem. Imaging 3 (2017), p. 15.

[6] C Ophus, Adv. Struct. Chem. Imaging 3 (2017), p. 13.

[7] AH Larsen et al., J. Phys. Condens. Matter Top. Rev. 29 (2017), p 273002.

[8] VP Pham and GY Yeom, Adv. Mater. 28 (2016), p. 9024.

[9] M Schleberger and J Kotakoski, 11 (2018), p. 1885.

[10] U Bangert et al., Nano Lett. 13(10) (2013), p. 4902.

[11] M Tripathi et al., ACS Nano 12 (2018), p. 4641.

[12] U Bangert et al., Ultramicroscopy 176 (2017), p. 31.

[13] JS Williams, Mater. Sci. Eng. A 253 (1998), p. 8.

[14] HE et al., Ultramicroscopy 133 (2013), p. 109.

[15] L Jones et al., Nano Lett. 14 (2014), p. 6336.

[16] This work is funded by the Irish Research Council Scholarship GOIPG/2015/2410.

(a)

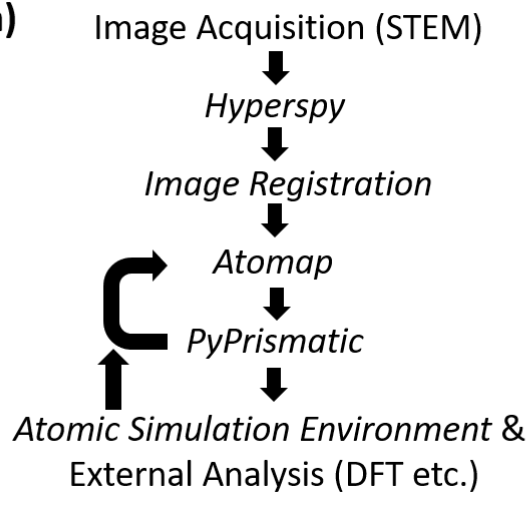

(b)

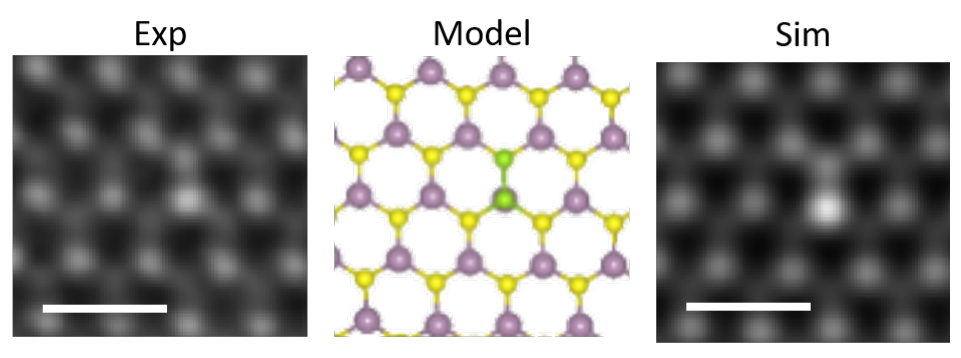

Figure 1. (a) Workflow incorporating several python packages for analysis of atomic resolution STEM images and image series. (b) Se implanted monolayer $\mathrm{MoS}_{2}$ experimental images are fed into the workflow from (a), automatically generating ball \& stick models and simulated images, allowing for further data analysis (e.g., DFT) of realistic atomic structures. Scale bar $0.5 \mathrm{~nm}$. 